\title{
La norme protectrice du divertissement : le droit du patrimoine culturel immatériel
}

Marie-Sophie de Clippele

Marie-Sophie de Clippele : Marie-Sophie de Clippele est aspirante F.S.R. - FNRS à l'Université Saint-Louis - Bruxelles / ENS Cachan. Elle y assure une série de séminaires portant sur l'actualité de la notion de 'communs' dans les matières portant sur la protection de l'environnement et de la culture, au départ de la dimension juridique mais dans une perspective d'élaboration commune et de partage de savoirs impliquant de nombreuses autres disciplines.

\section{DOI: $\underline{10.25518 / 1370-2262.284}$}

\section{Résumé :}

La sauvegarde des divertissements et du folklore d'antan, et encore vivants aujourd'hui, passe par le patrimoine culturel immatériel. Apparue récemment dans les différentes sources juridiques, la notion de patrimoine culturel immatériel est de plus en plus reconnue, même si de nombreuses questions et incertitudes demeurent.

Cet article vise à analyser l'historique de la notion à travers les sources juridiques, commençant par la sphère internationale, là où la reconnaissance du patrimoine culturel immatériel a germé et a été consacrée. S’inspirant du modèle international et contribuant au succès de la Convention de l'UNESCO de 2003, la législation en Belgique reconnait de plus en plus l'importance de la sauvegarde de ce patrimoine et constitue, notamment par sa diversité institutionnelle, un objet d'étude intéressant.

Mots-clés : Communautés, Convention pour la sauvegarde du patrimoine culturel immatériel (2003), Convention-cadre sur la valeur du patrimoine culturel pour la société (2005), Développement durable, Diversité culturelle, Expressions du folklore, OMPI, Patrimoine culturel immatériel, Politiques publiques culturelles, Protection juridique, Savoirs traditionnels, UNESCO

\begin{abstract}
:
Intangible cultural heritage is key in the safeguarding of ancient yet still vivid folklore and entertainment. The notion of intangible cultural heritage only appeared recently in the several sources of law. Its recognition increases over time, however certain questions and uncertainties remain.

This article aims to analyse the historical development of the notion through the sources of law. It first started in the international sphere, where the recognition of intangible cultural heritage germinated and was established under the auspices of UNESCO. Inspired by the international model and contributing to the success of the 2003 UNESCO Convention, Belgian legislation progressively recognized the importance of safeguarding this heritage. Because if its complex institutional scheme, Belgian legislation offers an interesting field of research.
\end{abstract}

\section{Introduction}

Les fêtes et divertissements d'antan se perpétuent parfois dans les villes et villages d'aujourd'hui. 
La norme protectrice du divertissement : le droit du patrimoine culturel imma...

L'on pense par exemple au Carnaval de Binche, au Carnaval d'Alost, à l'Ommegang de Bruxelles ou encore aux Géants et dragons processionnels de Belgique.

Ces divers événements festifs sont estimés représentatifs d'une communauté culturelle et, à ce titre, méritent d'être protégés en tant qu'éléments du patrimoine culturel immatériel. La démarche est ainsi toute autre que celle cherchant à édicter des normes qui limitent, censurent et sanctionnent les expressions culturelles populaires. Certes, en cas d'atteinte à l'ordre public, notamment à la propreté, la salubrité, la sûreté et la tranquillité dans les rues, lieux et édifices publics $\underline{1}$, le droit intervient comme contrôleur des pratiques sociales. Mais le prisme à travers lequel le droit actuel regarde ces divertissements est nouveau : il vise également à sauvegarder ces éléments.

L'enjeu est triple : social, politique et économique. Socialement, il s'agit de répondre à la menace de la mondialisation par la reconnaissance de la diversité culturelle et de l'importance du patrimoine immatériel en tant que porteur d'identité culturelle et vecteur de continuité de la communauté détentrice de ce patrimoine. Politiquement, la protection du patrimoine immatériel implique la reconnaissance de ses détenteurs voire de droits subjectifs en faveur de ces derniers, suscitant la crainte des États face à des revendications identitaires et communautaristes. Enfin, sur le plan économique, certains éléments disposent d'une valeur financière, pouvant faire l'objet d'échanges commerciaux. Il importe alors de protéger ces éléments contre des appropriations illicites ou des exploitations abusives souvent menées par le monde industriel peu sensible aux valeurs culturelles de ces éléments.

Si le droit cherche désormais à protéger ces éléments immatériels, la notion de "patrimoine culturel immatériel » n'est toutefois pas exempte de difficultés et d'incertitudes terminologiques. L'on songerait intuitivement plutôt au terme de «folklore». Mais auparavant, le folklore avait une connotation péjorative et s'inscrivait en opposition à la culture des pays développés. En effet, l'opposition sociale entre la création individuelle de l'élite bourgeoise et les traditions du peuple a pris une tournure nouvelle avec les colonisations, où la production artistique des sociétés occidentales se définit par opposition au folklore des communautés locales 2 . Depuis, la culture populaire a été reconnue comme catégorie patrimoniale à part entière et la culture dite "d'élite » y a même été insérée afin d'éviter que le patrimoine immatériel ne soit qu'une " expression tiersmondiste $» \underline{3}$.

D’autres termes comme «populaire », «traditionnel », « vivant», « oral » ou «propriété culturelle et intellectuelle » ont tour à tour été envisagés, mais abandonnés faute de comprendre l'ensemble du concept. Comme la plupart de ces notions, le terme «immatériel » ne rend pas compte de la totalité de ce patrimoine culturel, notamment de son rôle social, et risque de devenir une source de confusion. Janet BLAKE semble lui préférer le «patrimoine culturel oral et traditionnel », intégrant les «deux aspects fondamentaux de ce patrimoine » $\underline{4}$. La confusion semble encore plus grande lorsque certains francophones parlent de patrimoine culturel « intangible » en référence à la notion anglaise, mais sans se douter qu'en réalité ils se trompent dans l'utilisation de ce terme $\underline{5}$.

Quoi qu'il en soit, la notion de patrimoine culturel immatériel a été retenue dans les diverses sources et disciplines juridiques et non juridiques. Faisant son entrée dans le monde juridique sous l'égide de l'UNESCO par l'adoption de la Convention pour la sauvegarde du patrimoine culturel immatériel du 17 octobre 2003, le patrimoine culturel immatériel se définit comme suit :

L'historique de la notion à travers les sources juridiques fait l'objet de notre analyse, commençant 
par la sphère internationale, là où la reconnaissance du patrimoine culturel immatériel a germé et a été consacrée (I. A.). S'inspirant du modèle international et contribuant au succès de la Convention de 2003, la législation en Belgique reconnait de plus en plus l'importance de la sauvegarde de ce patrimoine et constitue, notamment par sa diversité institutionnelle, un objet d'étude intéressant (I.B.).

\section{Les sources internationales et européennes à la genèse du patrimoine culturel immatériel}

\section{Le cadre normatif de l'UNESCO et l'émergence du patrimoine culturel immatériel}

Principale représentante des organisations internationales à vocation culturelle, l’UNESCO constitue l'institution phare en vue d'assurer la protection du patrimoine culturel à laquelle elle œuvre depuis près de soixante ans $\underline{6}$.

\section{La prépondérance historique du patrimoine matériel}

Historiquement, l'UNESCO est fondée sur un « régime d'objet » $\underline{7}$, s'appuyant sur une conception du patrimoine héritée des civilisations européennesㅁ․ L’UNESCO a longtemps privilégié la protection des monuments, des sites et autres représentations matérielles du patrimoine et n'octroyait qu'une place secondaire et indirecte au patrimoine immatériel.

La Convention pour la protection du patrimoine mondial, culturel et naturel de 1972 (ci-après

« Convention de 1972 ») a en effet surtout été soucieuse de préserver l’aspect matériel du patrimoine et a veillé à la protection des œuvres présentant une «valeur universelle exceptionnelle » $\underline{9}$ et permettant une «diffusion mondiale du concept de patrimoine » $\underline{10}$. L'aspect immatériel n'a été pris en compte que depuis peu dans les Orientations, et cela de manière fort limitée11.

En réalité, le principe d'universalité et d'exceptionnalité du patrimoine mondial profita surtout aux pays occidentaux, riches en patrimoine bâti et en sites urbains. La répartition des biens inscrits témoigne encore aujourd'hui des déséquilibres à l'origine $\underline{12}$. Si d'autres formes de patrimoine ont petit à petit été consacrées, tels que les sites naturels ou le paysage culturel, force est de constater la limitation de ce qui est reconnu par la Convention de 1972 par rapport à la variété des approches patrimoniales au plan mondial13.

Les réactions des pays non occidentaux ne se sont pas faites attendre et, dès les années septante, les premières demandes de protection d'un patrimoine non matériel ont été émises 14 . L'enjeu politique dans les oppositions entre pays du Nord et du Sud était à peine caché, mais il y avait également un véritable vide patrimonial à combler.

\section{La prise en compte du patrimoine culturel immatériel dans divers instruments}

En réponse aux demandes des pays non occidentaux, les premiers instruments sont établis, mais ceux-ci ne reconnaissent que de manière secondaire le patrimoine culturel immatériel. Se déployant dans divers domaines, la notion de patrimoine culturel immatériel est alors prise en considération et s'inscrit dans un contexte plus large que le secteur culturel. 
La norme protectrice du divertissement : le droit du patrimoine culturel imma...

D'abord, les droits de l'homme ont évolué et accordent une importance grandissante pour les droits culturels, qui reconnaissent l'importance fondamentale de protéger le patrimoine culturel immatériel.

Ensuite, le patrimoine culturel immatériel est mentionné dans le droit des peuples autochtones et leur patrimoine autochtone $\underline{15}$. Compris de manière très large, ce patrimoine renvoie à tous les biens culturels meubles tels que définis par les conventions pertinentes de l'UNESCO; toutes les formes d'œuvres littéraires et artistiques dans les domaines de la musique, de la danse, des chants, des cérémonies, ainsi que des symboles et graphismes, des narrations et de la poésie; toutes les formes de connaissances scientifiques, agricoles, techniques et écologiques, y compris les cultigènes, les médicaments et l'utilisation rationnelle de la flore et de la faune; les restes humains; les biens culturels immeubles dont les sites sacrés, les sites d'importance historique et les lieux de sépulture; les enregistrements issus du patrimoine des peuples autochtones sous forme de films, de photographies, de vidéocassettes ou d'audiocassettes 16 . Les différents textes relatifs aux peuples autochtones et leur patrimoine17, bien que non contraignants, prévoient des droits culturels ainsi que des mesures de propriété intellectuelle concernant les connaissances autochtones. Toutefois, force est de constater un «traitement partiel, voire partial» de ces connaissances autochtones ainsi qu'une « standardisation inappropriée de la propriété intellectuelle comme modèle juridique de protection $» \underline{18}$, sans parler de l'absence de définition universelle du terme « peuple autochtone » 19 .

Le patrimoine culturel immatériel se retrouve également dans les thèmes liés au développement durable et à la biodiversité. Dans la mesure où le patrimoine culturel immatériel ne reflète pas seulement une construction artistique ou esthétique, mais peut apparaître comme un réel mode de vię20, il contribue au développement durable d'une société donnée. Les connaissances traditionnelles représentent le mieux cette vision intersectorielle car elles constituent un élément essentiel du « capital social » $\underline{21}$ d'une communauté, exprimant ses relations sociales, ses valeurs socioculturelles et sa conception du monde. Dès lors que 70 à $80 \%$ de la population africaine recourt aux médecines traditionnelles pour des soins de santé primaires, l'importance de la valeur sociale mais aussi économique des connaissances traditionnelles est considérable, celle-ci devant s'inscrire dans un processus de développement durable adapté aux concepts socioculturels spécifiques $\underline{22}$.

Concernant plus précisément la biodiversité, la Convention de 199223 vise à favoriser la conservation in situ et prévoit en son article 8 (j) la protectiondes connaissances, innovations et pratiques des communautés autochtones et locales tant par la participation et le consentement préalable de la communauté que par le partage équitable des avantages 24 . Les aspects de propriété intellectuelle et les dispositions correspondantes traitant de l'accès et du partage des avantages dans le domaine des ressources génétiques ont été confiés à l'OMPI en tant qu'organisation spécialisée dans ce domaine25. Cette collaboration entre les Nations Unies et l'OMPI pourrait constituer un possible modèle pour l'UNESCO dans le domaine du patrimoine culturel immatériel, malgré une tentative infructueuse dans le passé.

Enfin, le patrimoine culturel immatériel s'inscrit dans la diversité culturelle, considérée comme son « creuset » $\underline{26}$. Cela est souligné dans la Déclaration universelle de l’UNESCO sur la diversité culturelle de 2001, dans la Déclaration d'Istanbul de 2002 et, de manière contraignante, dans la Convention sur la promotion de la diversité des expressions culturelles de 2005. En tant que telle, la diversité culturelle constitue un «patrimoine commun de l'humanité », fondamentalement 
《inhérente » $\underline{27}$ à celle-ci. Décrite encore comme une diversité qui s'incarne dans «l'originalité et la pluralité des identités ainsi que dans les expressions culturelles des peuples et des sociétés qui constituent l'humanité », on comprend mieux l'importance de son respect dans le contexte de mondialisation, parfois perçu comme une menace d'uniformisation28. Visant à reconnaître la double nature des biens et services cultures tant économiques que culturels $\underline{29}$, la diversité culturelle constitue également un des objectifs du patrimoine culturel immatériel qui est confronté à la même question de double reconnaissance.

\section{Les premières tentatives de reconnaissance du patrimoine immatériel}

La première consécration à part entière du patrimoine culturel immatériel a été établie en 1989 par la Recommandation sur la sauvegarde de la culture traditionnelle et populaire, qui constitue une « innovation conceptuelle significative » $\underline{30}$.

La Recommandation se penche particulièrement sur la protection du folklore comme faisant « partie intégrante du patrimoine culturel et de la culture vivante » et précise son importance pour l'identité sociale et culturelle. La définition donnée par la Recommandation met déjà l'accent sur la communauté disant que la culture traditionnelle et populaire ( folklore » dans le texte anglais) est l'ensemble des créations émanant d'une communauté culturelle fondées sur la tradition, exprimées par un groupe ou par des individus et reconnus comme répondant aux attentes de la communauté en tant qu'expression de l'identité culturelle et sociale de celle-ci31. Mais, outre le fait que la définition ne tient pas compte du contexte social, culturel et intellectuel de la création et du maintien du folklore, les communautés figurent uniquement comme objet du folklore, le rôle d'identification incombant aux chercheurs scientifiques $\underline{32}$. De même, la «conservation de la culture traditionnelle et populaire $\$ \underline{3}$ repose entre les mains de la communauté scientifique par l'établissement d'inventaires et autres mesures de documentation. D'autres sections abordent la préservation, la diffusion et la protection de la culture traditionnelle et populaire, laissant aux États la responsabilité de mise en œuvre.

Mais les États sont timides dans la mise en pratique de la Recommandation, celle-ci souffrant par ailleurs d'un manque de mandat spécifique de l'UNESCO, voire d'un manque général de mécanismes internationaux $\underline{34}$. Les obligations de sauvegarde imposées aux États membres ne sont accompagnées d'aucune directive, laissant ces derniers hésitants quant aux types de mesures à prendre en vue de sauvegarder ce patrimoine $\underline{35}$. Les États sont souvent réticents à protéger un patrimoine culturel et préfèrent laisser le champ libre au développement économique et, plus largement, à la mondialisation.

\section{La consécration conventionnelle du patrimoine immatériel}

Premier instrument contraignant pour la sauvegarde du patrimoine culturel immatériel, la Convention de 2003 connaît un succès incontestable. Le rythme de ratifications reste en effet « impressionnant » $\underline{6} 6$ et le nombre de dossiers introduits pour inscription a un taux tellement élevé que la $4{ }^{\text {ème }}$ AG en juin 2012 a décidé d'insérer dans les Directives opérationnelles un plafond audelà duquel les demandes ne seront plus examinées par le Comité37.

En ce qui concerne la notion de patrimoine culturel immatériel, la Convention opta pour une définition « complexe » $\underline{38}$ en essayant de garder une formule suffisamment synthétique et facilement applicable sur le plan opérationnel. Ainsi, selon l'article 2, le patrimoine culturel 
La norme protectrice du divertissement : le droit du patrimoine culturel imma...

immatériel comprend

les pratiques, représentations, expressions, connaissances et savoir-faire - ainsi que les instruments, objets, artefacts et espaces culturels qui leur sont associés - que les communautés, les groupes et, le cas échéant, les individus reconnaissent comme faisant partie de leur patrimoine culturel.

Ce patrimoine culturel immatériel, transmis de génération en génération, est recréé en permanence par les communautés et groupes en fonction de leur milieu, de leur interaction avec la nature et de leur histoire, et leur procure un sentiment d'identité et de continuité, contribuant ainsi à promouvoir le respect de la diversité culturelle et la créativité humaine. Aux fins de la présente Convention, seul sera pris en considération le patrimoine culturel immatériel conforme aux instruments internationaux existants relatifs aux droits de l'homme, ainsi qu'à l'exigence du respect mutuel entre communautés, groupes et individus, et d'un développement durable.

C'est précisément dans la définition du concept que réside un des acquis majeurs de la Convention. En rassemblant différentes catégories patrimoniales, le concept de patrimoine culturel immatériel leur confère une « unité nouvelle » et crée un « effet de masse » profitable à tout un ensemble d'objets patrimoniaux auparavant dispersés $\underline{39}$.

La mise en œuvre de la Convention repose sur l'obligation «fondamentale » 40 pour les États Parties de sauvegarder le patrimoine culturel immatériel dans l'intérêt général de l’humanité41. Pour ce faire, la Convention de 2003 reprend, mutatis mutandis, l'approche générale de la Convention de 1972 et précise les obligations des États Parties au niveau national 42 ainsi qu'au niveau international $\underline{43}$. De même, le mécanisme des listes, des programmes et des projets ainsi que la mise en place des organes reposent sur la logique patrimoniale de la Convention de 1972.

Néanmoins, son but de sauvegarde lui permet de se démarquer de l'objectif de conservation de la Convention de 1972. En d'autres termes, la notion d'exceptionnalité antérieurement mise en avant pour le patrimoine mondial est remplacée par la notion de représentativité, excluant toute idée de hiérarchie entre les éléments du patrimoine culturel immatériel au demeurant incompatible avec

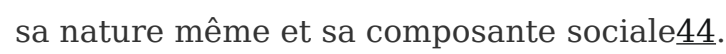

Cependant, si la Convention de 2003 s'attache à sauvegarder le patrimoine culturel immatériel, elle a délibérément contourné les questions relatives aux droits de propriété intellectuelle et/ou culturelle, ce qui lui laisse un goût d'inachevé.

\section{L'Organisation mondiale de la propriété intellectuelle (OMPI) et la protection juridique du patrimoine culturel immatériel}

Suite au développement des biotechnologies modernes, la valeur économique, scientifique et commerciale des ressources génétiques a augmenté et, par conséquent, les savoirs traditionnels associés à ces ressources génétiques ont attiré l'attention d'un plus large public. D'autres créations fondées sur la tradition, comme les expressions du folklore, ont parallèlement acquis un poids économique et culturel nouveau dans une société de l'information mondialisée $\underline{45}$.

Dès lors, les questions de propriété intellectuelle relatives à la conservation, à la gestion, à l'utilisation durable et au partage des bénéfices pour les ressources génétiques et les savoirs traditionnels qui leur sont associés ont gagné en importance, particulièrement pour les pays en voie de développement, souvent très riches en ces domaines. 
Dans la mesure où certains éléments du patrimoine culturel immatériel, comme les savoirs traditionnels et les expressions du folklore $\underline{46}$, représentent un enjeu économique croissant, il revient à l'OMPI en tant qu'institution spécialisée des Nations Unies dans la promotion de la propriété intellectuelle, de se pencher sur la question des échanges économiques et de la protection juridique de ces éléments du patrimoine immatériel. Il s'agit de prendre en charge la protection juridique contre toute appropriation illicite ainsi que l'accès et le partage équitable des avantages découlant de la commercialisation des savoirs traditonnels et expressions du folklore.

L'OMPI vise seulement à protéger des droits subjectifs des communautés et, en cela, protège de manière plus restreinte le patrimoine culturel immatériel que la Convention de 2003. Cette dernière cherche en effet à sauvegarder l'ensemble des éléments du patrimoine immatériel et non pas seulement les éléments qui peuvent être exploités commercialementㅆ7.

À cette fin, l'OMPI a créé le Comité intergouvernemental de la propriété intellectuelle relative aux ressources génétiques, aux savoirs traditionnels et au folklore (ci-après, « IGC »)쓰. L'IGC constitue le cadre dans lequel les États membres peuvent poursuivre leurs échanges de vue sur ces trois thèmes principaux 49 . Jusqu'à présent, l'IGC s'est réuni plusieurs fois par an, la $28{ }^{\text {ème }}$ session ayant eu lieu du 7 au 9 juillet 2014무. Il a déjà élaboré un nombre considérable de documents, sans qu'aucun n'ait toutefois obtenu un statut officiel. Le travail de l'IGC est particulièrement remarquable car il a rassemblé un nombre considérable d'experts et de représentants de communautés autochtones ou locales, afin de réunir les différents points de vue dans un débat à intérêts divergents.

\section{Le droit européen et la nouvelle notion du patrimoine culturel}

Si les sphères internationales se préoccupent de plus en plus du patrimoine culturel immatériel, tel n'est pas encore le cas au niveau du droit européen. Tant le droit de l'Union européenne que celui du Conseil de l'Europe ont encore relativement peu traité ce domaine, car ayant du mal à trouver un « champ d'ouverture » $\underline{51}$ sur l'immatériel. Les récents développements indiquent cependant une plus grande prise en compte.

Concernant le droit du Conseil de l'Europe, celui-ci s'est longtemps préoccupé du patrimoine matériel57. Récemment, le Conseil de l’Europe s'est cependant intéressé à la question du patrimoine immatériel, et plus largement, à la notion de patrimoine culturel. En effet, après dix années de préparation $\underline{58}$, le Conseil de l’Europe a adopté la Convention-cadre sur la valeur du patrimoine culturel pour la société, rédigée à Faro le 27 octobre 2005. Tranchant de manière nette avec la lecture dichotomique du patrimoine matériel ou immatériel, ce texte propose une nouvelle approche de la valeur du patrimoine culturel. Selon la Convention-cadre, le patrimoine culturel n'est pas une fin en soi, mais contribue au bien-être des personnes et des attentes de la société $\underline{59}$. Sa valeur se décline comme une ressource de développement durable et de qualité de vie dans une société en constante évolution $\underline{60}$. L’approche se veut ainsi fonctionnelle, complétant la perception esthétique et académique du patrimoine culturel.

Bien que la Convention-cadre ne soit qu'un texte de nature philosophique, éthique et déontologique sans caractère contraignant, elle constitue une forte source d'inspiration pour ses États membres $\underline{61}$.

Perçu de manière holistique, le patrimoine culturel constitue un ensemble de ressources héritées du passé que des personnes considèrent, par-delà le régime de propriété des biens, comme un reflet et une expression de leurs valeurs, croyances, savoirs et traditions en continuelle évolution. 
La norme protectrice du divertissement : le droit du patrimoine culturel imma...

Cela inclut tous les aspects de l'environnement résultant de l'interaction dans le temps entre les personnes et les lieux. Sans préciser les différentes composantes du patrimoine culturel, la définition donne un puissant signal d'une nouvelle évolution de la notion de patrimoine culturel non pas sectorielle mais globale. Serait-ce le premier pas vers une reconnaissance internationale d'une vision intégrée et complète du patrimoine culturel ?

Tout comme la définition très large de l'objet, le sujet du patrimoine culturel reçoit une définition englobante au travers du concept de «communauté du patrimoine » $\underline{62}$, qui est intéressant en vue de préciser et d’appréhender le concept de communautés, groupes et individus dans le cadre du patrimoine culturel immatériel.

La Convention-cadre est également le premier texte à reconnaître l'existence du droit au patrimoine culturel $\underline{63}$, découlant du droit de participer à la vie culturelle.

\section{La mise en ouvre du patrimoine culturel immatériel : le droit belge précurseur}

La mise en œuvre du concept de patrimoine culturel immatériel en droit interne dépend de chaque structure étatique et de l'importance que les institutions et politiques publiques cherchent à accorder à ce patrimoine. Le droit belge, contrairement à la majorité des pays, reconnaît depuis plus longtemps l'importance du patrimoine culturel immatériel, lui offrant même un cadre légal.

\section{La reconnaissance constitutionnelle des communautés}

Dès les années septante, l'État belge a reconnu un statut juridique autonome aux trois communautés selon leur spécificité culturelle, fondée notamment sur la langue, et a entamé le processus de fédéralisation. Ainsi, la Communauté flamande, la Communauté française (dénommée « Fédération Wallonie-Bruxelles » depuis 2011 pour les communications usuelles $\underline{64}$ ) et la Communauté germanophone sont devenues des personnes morales de droit public dotées d'une autonomie constitutive totale et couvrant l'entièreté du territoire belge.

En 1994, la structure fédérale de l'État a formellement été reconnue par la Constitution, répartissant le territoire en trois communautés pour certains blocs de compétences et en trois régions $\underline{65}$ pour d'autres. L'État fédéral demeure compétent pour les matières résiduaires. Cette structure étatique reflète une vision différentialiste et libérale de la société 66 et offre une reconnaissance constitutionnelle des communautés ainsi que de sa capacité à déterminer son identité et son patrimoine propre.

Basées sur le principe de personnalité, les Communautés sont en principe compétentes dans les matières culturelles. En effet, au lendemain de la fédéralisation de l’État belge, la compétence en matière culturelle a été transférée aux Communautés en vertu des articles 127 , § $1^{\mathrm{er}}$ et 130 , § $1^{\mathrm{er}}$ de la Constitution. Celles-ci sont alors devenues compétentes, en vertu de la loi du 21 juillet 1971, pour légiférer, entre autres, dans le domaine du patrimoine culturel. Compris comme couvrant un « large domaine $\underline{67}$ qui « désigne le patrimoine culturel tant mobilier qu'immobilier » $\underline{68}$ ainsi que le patrimoine immatériel (alors souvent dénommé folklore ou culture populaire), il inclut par exemple la réglementation relative à l'exportation d'œuvres d'art, au dépôt légal, à la conservation des monuments, sites et lieux présentant un intérêt historique, etc. 69. 
En 1988, toutefois, la compétence des monuments et des sites fut transférée aux Régions $\underline{70}$, dissociant ainsi le patrimoine culturel immobilier du patrimoine culturel mobilier. Cette séparation permet, d'une part, une politique plus coordonnée dans les domaines de l'aménagement du territoire, de l'urbanisme, des monuments et des sites, de la sorte réunis entre les mains d'une seule autorité, mais, d'autre part, présente l'inconvénient de provoquer une «scission absolue » entre le patrimoine mobilier et immobilier qui risque d'être ressentie comme artificielle et inefficace $\underline{71 .}$ Désormais, le patrimoine mobilier reste l'apanage des Communautés, avec une réserve en faveur de l'État fédéral compétent pour les «établissements scientifiques et culturels fédéraux » $\underline{72}$ dont la liste est établie par arrêté royal73. Le patrimoine immatériel demeure également sous la compétence des Communautés, la Communauté française ayant été la première collectivité territoriale en Europe à lui offrir une protection légale et la Communauté flamande menant une politique très active et avancée dans le domaine.

Le contexte politique et juridique permet de mieux comprendre en quoi l'expérience belge en matière patrimoniale est intéressante et constitue une source d'inspiration pour d'autres États $\underline{74}$. La Belgique a ratifié la Convention de 2003, après que celle-ci ait été adoptée par les trois Communautés, le 24 mars 2006.

\section{Le décret de la Communauté française du 11 juillet 200275}

Le décret de la Communauté française est le premier texte en Europe à élaborer un outil législatif de protection du patrimoine culturel immatériel, après s'être inspiré des textes du Japon $\underline{76}$ et d'autres pays d'Asie. De manière originale et même avant-gardiste, ce décret regroupe la protection du patrimoine mobilier et immatériel, perçus comme complémentaires et indissociables, malgré leur différente logique fonctionnelle77. En cela, l'expérience belge prouve qu'il est possible pour un pays occidental de légiférer pour protéger le patrimoine immatériel de la même manière que pour le patrimoine mobilier ou immobilier $\underline{\text { i8. }}$

Concernant le patrimoine immatériel, les experts belges ont étroitement collaboré aux travaux de l’UNESCO79. Par conséquent, le décret s'inspire des notions du programme de l'UNESCO pour les trésors humains vivants et du programme pour les chefs-d'œuvre du patrimoine oral et immatériel, reprenant presque les mêmes termes $\underline{80}$.

Le patrimoine immatériel, tel que défini à l'article 26 du décret, est composé de trois éléments principaux: les personnes physiques pouvant obtenir le titre de «trésor culturel vivant de la Communauté française» $\underline{81}$; les manifestations recevant le titre de « chef-d'œuvre du patrimoine oral et immatériel de la Communauté française $\unrhd \underline{82}$ et les lieux culturels physiques devenant « espace du patrimoine oral et immatériel de la Communauté française » $\underline{83}$. Cette répartition tripartite permet une reconnaissance plus complète et plus détaillée que celle prévue dans le décret du 26 mai 1981 instituant un Conseil supérieur des Arts et Traditions populaires. Les critères de protection pour les chefs-d'œuvre se retrouvent dans l'article 28 du décret, laissant au Gouvernement le soin de les préciser, mais devant au moins comprendre :

1. le fondement de la création sur la tradition;

2. l'expression par un groupe ou par des individus ;

3. la reconnaissance de la manifestation par la communauté comme répondant aux attentes de celle-ci en tant qu'expression de son identité culturelle et sociale; 
La norme protectrice du divertissement : le droit du patrimoine culturel imma...

4. la transmission des normes et des valeurs oralement, par imitation ou par d'autres manières.

L'article 15 de l'Arrêté du Gouvernement de la Communauté française reprend les mêmes critères pour que les manifestations obtiennent le titre de chef-d'œuvre, en précisant au surplus que :

$5^{\circ}$ elle est conforme aux principes des Droits de l'Homme ;

$6^{\circ}$ elle recouvre au moins une des activités humaines touchant aux domaines suivants :

a) Formes d'expressions orales;

b) Fêtes, spectacles et jeux traditionnels ;

c) Artisanat et savoir-faire ;

d) Connaissances et pratiques traditionnelles concernant la nature, dont l'agriculture et l'alimentation ;

e) Rites et coutumes.

En outre, la manifestation peut remplir les critères suivants :

$1^{\circ}$ elle est constamment recréée par la communauté dont elle est issue en fonction du milieu et de l'histoire de celle-ci ;

$2^{\circ}$ elle procure aux individus et aux groupes qui la pratiquent un sentiment de continuité et d'identité ;

$3^{\circ}$ elle présente des qualités esthétiques $\underline{84}$.

Les deux autres volets de la protection immatérielle ne disposent toutefois pas de critères précis. Le décret renvoie la tâche au Gouvernement, mais ce dernier n'a pris que des dispositions de nature procédurale pour les trésors vivants $\underline{85}$ et aucune mesure spécifique pour les espaces du patrimoine oral et immatériel.

$\mathrm{Au}$ niveau institutionnel, le décret met en place la Commission consultative du Patrimoine oral et immatériel (article 3) chargée de proposer la reconnaissance des chefs-d'œuvre, des trésors culturels vivants et des espaces du patrimoine oral et immatériel ainsi que d'examiner des demandes de subventions et de préparer l'introduction des candidatures à l'UNESCO $\underline{86}$. Complétant l'action de la Commission, une autre instance d'avis a été créée en 2007 - le Conseil d'ethnologie 87 - en vue de s'occuper des études, enquêtes et publications dans le domaine du patrimoine immatériel.

Le travail de la Commission est assez positif, cette dernière ayant déjà émis de nombreuses propositions pour des manifestations, mais non pour les deux autres composantes. Malgré un relatif succès au niveau interne (trente-neuf chefs-d'œuvre reconnus $\underline{88}$ ) et à l’UNESCO (cinq dossiers acceptés), Jean-Pierre DuCASTELLE, président de la Commission, déplore un « absentéisme chronique » $\underline{89}$ de certains membres de la Commission siégeant par ailleurs dans d'autres instances et dont le domaine de compétence ne coïncide qu'en partie avec celui du patrimoine immatériel. En outre, aucun inventaire scientifique n'a pu être mis en œuvre, alors que la documentation est importante. Certaines études devraient donc être menées par le Conseil d'ethnologie avant 
d'émettre un avis sur des demandes de reconnaissance et d'inscription. Enfin, le budget, bien que relativement maigre, n’a pas toujours été mobilisé $\underline{90}$, par manque d'information et vu le nombre limité de chefs-d'œuvre, ce qui pourrait évoluer les prochaines années.

\section{La politique culturelle immatérielle de la Communauté flamande}

Contrairement à son homologue francophone, la Communauté flamande n'a pas rédigé d'outil législatif spécifique portant sur le patrimoine culturel immatériel. La notion de patrimoine culturel immatériel n'est inclue dans les textes légaux que depuis le décret sur le Patrimoine culturel de 2008, révisé en 201291, lorsque le Décret de 1997 sur la Culture populaire a été intégré dans le Décret de 2008 sur le Patrimoine Culturel et que la définition de patrimoine culturel a repris le versant immatérielg2, s'inspirant de la Convention-cadre du Conseil de l'Europe de 2005.

En 2008, fut également élaboré un règlement de nature politique93 afin de créer un « Inventaire du patrimoine culturel immatériel en Flandre " $\underline{94}$, comprenant les critères et procédures pour placer un élément sur cet inventaire. Les critères imposent que :

1. l'élément réponde à la définition de patrimoine culturel immatériel telle que reprise dans la note de vision ;

2. les communautés du patrimoine aient convenu par consensus d'inscrire cet élément sur l'inventaire. Ce consensus est démontré par l'implication des groupes et communautés et éventuellement des individus faisant partie des communautés du patrimoine ;

3. la communauté du patrimoine soit active dans la transmission du patrimoine culturel immatériel. Ceci est démontré par des actions émises et à émettre dans la communauté ;

4. une organisation du patrimoine culturel subsidiée par la politique patrimoniale 95 est impliquée dans la communauté et la soutient dans la sauvegarde de son patrimoine culturel immatériel. La sauvegarde du patrimoine culturel immatériel comprend l'identification, la qualification, la documentation, la recherche et la transmission de ce patrimoine.

Ce règlement comprend également plusieurs contrôles de qualité. D'une part, il demande à ce que la communauté du patrimoine $\underline{6}$ collabore avec une organisation subventionnée sur la base du Décret sur le Patrimoine Culturel de 2008. D'autre part, le décret cherche à ce que la Commission du patrimoine culturel ad hoc, créée par le Ministre de la Culture, examine la qualité du dossier et offre des conseils à la communauté et au Ministre de la Culture sur l'intégration éventuelle de ce dossier dans l'Inventaire en Flandre et/ou devant l'UNESCO.

Toutefois, le règlement ne saurait remplacer un réel décret, nécessaire pour déterminer le montant des subsides ainsi qu'une politique pour les ONG, bien plus dirigée vers les institutions et organisations. Par ailleurs, la Commission ad hoc peut ne pas être renouvelée si le Ministre n'en voit pas l'utilité, laissant le travail de la Commission ad hoc dans l'insécurité juridique.

Outre la Commission ad hoc, l'interface flamande pour le patrimoine culturel (FARO)g7 ayant intégré le Centre flamand pour la Culture populaire (VCV) est très active dans le domaine. L'association sans but lucratif (asbl) « Tapis plein » collabore également avec les communautés et diverses instances afin de faciliter le travail de sauvegarde et d'inventorisation. L'Agence des Arts et du Patrimoine, division du Ministère de la culture, de la jeunesse, du sport et des médias de la 
La norme protectrice du divertissement : le droit du patrimoine culturel imma...

Communauté flamande, constitue le relais institutionnel et supervise par ailleurs l'interface FARO.

Comblant le manque de mesures légales, la Communauté flamande poursuit une "politique de facilitation $\underline{98}$ très active dans le domaine du patrimoine culturel. La Communauté a même rédigé une note de vision détaillée concernant le patrimoine immatérielg9, qui précise d'emblée que la Flandre prône une vision holistique du patrimoine culturel selon un rapport trinitaire entre patrimoine mobilier, immobilier et immatériel. La philosophie poursuivie par la politique culturelle flamande est de pouvoir offrir aux communautés les opportunités de transmission.

\section{Les timides protections en Communauté germanophone}

Troisième entité fédérée compétente en matière de patrimoine immatériel, la Communauté germanophone n'a cependant pas encore pris de mesures législatives (un projet de décret créant un inventaire des éléments du patrimoine culturel immatériel et promouvant les mesures de sauvegarde de ce patrimoine est préparé, le «Bewahrung des immateriellen Kulturerbes »100, mais il n'est pas encore mis à l'agenda du Parlement de la Communauté germanophone), ni élaboré de politique publique à ce sujet.

Néanmoins, elle a mis sur pied un projet de développement régional visant notamment la sauvegarde des dialectes $\underline{101}$ et des traditions $\underline{102}$.

\section{Conclusion}

De l'analyse des différentes sources et composantes du patrimoine culturel immatériel, force est de constater la transformation dans le droit du patrimoine culturel. Les changements traduisent un passage du droit de propriété sur les choses, fondé sur l'intérêt public, à un droit des personnes à ces choses, fondé sur le lien d'appartenance à la communauté103. Le centre de gravité du droit bascule du bien à la personne, individuelle ou collective, partant du principe qu'en protégeant le lien que la personne entretient avec ses ressources culturelles, celles-ci seront protégées. Des traces de ce renversement de perspective se retrouvent déjà dans la Convention-cadre où la nécessité de placer « la personne et les valeurs humaines au centre d'un concept élargi et transversal du patrimoine culturel »104 est pointée.

Si l'objet de cet article n'a pas été d'approfondir les questions afférentes au droit du patrimoine culturel immatériel, il nous semble intéressant de mettre en lumière certaines d'entre elles soulevées durant notre recherche.

En effet, le passage du droit des choses au droit des personnes à ces choses pose la question de l'objet de ce droit des personnes. S'agirait-il d'un droit de propriété culturelle, octroyant une protection pécuniaire contre les exploitations illicites? Mais, ce droit ne serait-il pas dès lors une résistance farouche du paradigme existant face au modèle rival ? La protection sui generis élaborée pour répondre aux lacunes du régime classique ne serait-elle pas une «hypothèse ad hoc $\gg \underline{105}$, bricolée par le système existant qui tente de garder le principe de la propriété au centre de la protection du patrimoine culturel ? Certes, la propriété culturelle présente de nombreux avantages en garantissant une protection absolue à son propriétaire privé, public ou collectif. Déjà là, toutefois, le problème se pose de vouloir allier propriété et dimension collective et révèle l'ébranlement du modèle. Le malaise du droit invite à imaginer des systèmes complémentaires ou adaptés, mais, à notre sens, ceux-ci s'accumulent au point de remettre en question le système 
en lui-même et d'ouvrir la voie au nouveau paradigme. Ainsi, certaines modalités permettent la protection du patrimoine culturel sans appropriation. Le droit moral ou la responsabilité civile se réfèrent aux droits de la personnalité, dans la logique de ce nouveau paradigme. Encore faut-il voir dans quelle mesure il peut accorder une protection pécuniaire ...

Le droit des personnes serait-il alors constitué de droits culturels ? Ce domaine est bien moins développé que celui de la propriété, très détaillé suite aux revendications des peuples autochtones. Les droits culturels sont encore en formation, mais il est possible d'en dégager des droits propices à la protection du patrimoine culturel. Si le droit d'accès au patrimoine et le droit à l'identité culturelle permettent d'affirmer une protection au patrimoine culturel immatériel, il en va plus fondamentalement du droit au patrimoine culturel106. Ce droit fondamental au patrimoine culturel pourrait offrir aux détenteurs du patrimoine immatériel une base juridique future qui rendrait compte de manière plus complète et ouverte de leurs attentes et besoins au niveau culturel. Ce faisant, le droit au patrimoine culturel et le droit des personnes s'inscrivent dans une logique de pluralité juridique. Tout comme les droits culturels, ils proposent une nouvelle approche du droit sous la bannière du « pluralisme constructif » 107 .

La diversité des sociétés et des droits suppose une pluralité des systèmes juridiques, perçue comme une « complémentarité des différences »108. Le droit international doit en effet promouvoir le «dialogue entre les cultures »109, sans gommer les différences ni s’y perdre. La logique du pluralisme en droit international implique une décentralisation du droit, où le droit international est subsidiaire aux droits nationaux, locaux ou coutumiers. Il convient alors de mettre en place un partenariat entre les organisations et les programmes internationaux, d'une part, et les divers instruments et ordres juridiques, d'autre part. La Convention de 2003 poserait-elle un premier jalon vers le pluralisme juridique et la diversité culturelle ? Son caractère contraignant ouvre la voie à une évolution affirmée en ce sens.

Cependant, beaucoup d'aspects restent à préciser et des obstacles s'érigent dans la pratique 110. Les communautés patrimoniales ne disposent souvent pas de la personnalité juridique ni des droits qui y sont rattachés et les États semblent peu enclins à leur accorder ce statut et ces droits. Même si les communautés étaient reconnues en tant que sujets de droits, l'effectivité de ces droits est loin d'être garantie. Le droit au patrimoine culturel se dessine petit à petit dans la doctrine, mais sa faible juridicité ne lui permet pas encore de protéger réellement les communautés et leur patrimoine culturel. Les droits personnels sans appropriation disposent, quant à eux, d'une juridicité et peuvent être invoqués devant les tribunaux, mais leur protection n'est que partielle car elle ne tient pas compte de la protection pécuniaire de ce patrimoine culturel immatériel. La trajectoire est encore parsemée d'embûches, surtout dans sa mise en pratique.

Il nous est néanmoins permis de voir l'avenir avec espoir, surtout après avoir analysé la situation belge qui détient un rôle d'exemple vis-à-vis des autres États en matière de protection du patrimoine immatériel111.

\section{Notes}

1 «Il s'agit de l'ordre public dit «matériel », qui ne recouvre pas l'ordre public moral (C.E., 11 janvier 2007, n 166.573, L\&S Vending). En revanche, depuis sa modification par la loi du 24 juin 2013 relatives aux sanctions administratives communales (entrée en vigueur le 1er janvier 2014), l'art. $135, \S 2$, al. $2,7^{\circ}$, de la Nouvelle loi communale recouvre également les mesures nécessaires 
La norme protectrice du divertissement : le droit du patrimoine culturel imma...

" afin de combattre toute forme d'incivilités ». Avant cette modification, cette disposition faisait référence à la notion de « dérangement public », dont la portée incertaine a été critiquée (A. MASSET, Les sanctions administratives dans les communes : en route vers le droit communal armé, J.T., 2001, p. 837). Selon la circulaire du 22 juillet 2014 explicative de la nouvelle réglementation relative aux sanctions administratives communales (M.B., 8 août 2014, p. 58140), le concept d'incivilités est identique à celui de dérangement public et vise « des comportement matériels, essentiellement individuels, qui sont de nature à troubler le déroulement harmonieux des activités humaines et à réduire la qualité de la vie des habitants d'une commune, d'un quartier, d'une rue d'une manière qui dépasse les contraintes normales de la vie sociale ». La circulaire énumère des exemples de ces « formes légères de troubles à la tranquillité, à la sécurité, à la salubrité et à la propreté publique » », M. VANDERSTRAETEN, La liberté d'entreprendre dans la jurisprudence de la Cour constitutionnelle et du Conseil d'État, dans Actualités en droit économique - La liberté d'entreprendre ou le retour en force d'un fondamental du droit économique, coll. UB ${ }^{3}$, Bruxelles, Bruylant, 2015, p. 29 et la note infrapaginale 111.

2 V. Tr. HALFSTEIN, Célébrer les différences, renforcer la conformité, dansCh. BORTOLOTTO (sous la dir.), Le patrimoine immatériel, Enjeux d'une nouvelle catégorie, Paris, Éditions de la Maison des sciences de l'homme, 2011, p. 81-83 (Ethnologie de la France cahier 26).

$\underline{3}$ L. WANG, La Convention pour la sauvegarde du patrimoine culturel immatériel et son application en droits français et chinois, thèse pour le doctorat en droit sous la direction de Jérôme Fromageau, Université Paris-Sud XI, Faculté Jean Monnet Droit Economie Gestion, 14 juin 2010, p. 69.

4 J. BLAKE, Élaboration d'un nouvel instrument normatif pour la sauvegarde du patrimoine culturel immatériel. Éléments de réflexion, éd. révisée, UNESCO, 2001, p. 10.

$\underline{5}$ C. KHAZNADAR précise que le terme « intangible » en anglais veut dire : «impalpable », alors qu'en français « intangible » veut dire « qu'on doit laisser intact, à quoi l'on ne doit pas toucher, immuable », C. KHAZNADAR, Avant-propos, dans Le patrimoine culturel immatériel : premières expériences en France, Paris, Actes Sud, 2011, p. 11-12 et note de bas de page (Internationale de l’Imaginaire, Nouvelle série, $n^{\circ}$ 25, Maison des cultures du monde, Babel, $n^{\circ} 1034$ ).

6 - Célébrations des 40 ans de la Convention sur le patrimoine mondial en 2012 à l'UNESCO, sous le thème du "Patrimoine mondial et développement durable : le rôle des communautés locales ", http://whc.unesco.org/fr/activites/664/, consulté le 3 avril 2015 ;

7 Ch. Bortolotto, Le trouble du patrimoine culturel immatériel, Terrain [En ligne], Le patrimoine culturel immatériel, Sommaire, mis en ligne le 15 novembre 2011, URL : http://terrain.revues.org/ 14447, p. 21.

8 Ch. HotTin, Une nouvelle perception du patrimoine, dansCh. HotTin (coordonnateur), Le patrimoine culturel immatériel. Culture et Recherche, n 116-117, printemps-été 2008, p. 15.

9 Convention pour la protection du patrimoine mondial, culturel et naturel, articles 1 et 2 et Orientations, para. 49 dans leur dernière version (2011) : « La valeur universelle exceptionnelle signifie une importance culturelle et/ou naturelle tellement exceptionnelle qu'elle transcende les frontières nationales et qu'elle présente le même caractère inestimable pour les générations actuelles et futures de l'ensemble de l'humanité. A ce titre, la protection permanente de ce 
patrimoine est de la plus haute importance pour la communauté internationale toute entière. Le Comité définit les critères pour l'inscription des biens sur la Liste du patrimoine mondial. » Toutefois, la notion de « valeur universelle exceptionnelle », clé de voûte de la Convention de 1972, suscite des divergences d'interprétation qui ont été remarquées dans le Rapport final de l'Audit de la Stratégie globale et de l'initiative PACTe (WHC-11/35.COM/INF.9A) relatives notamment à la variation dans l'espace et dans le temps (p. 8-10 et 14-16 du Rapport).

10 Ch. HotTin, Une nouvelle perception du patrimoine, op. cit., p. 15.

11 - Orientations, para. 77, énumère les différents critères pouvant être pris en compte en vue de reconnaître la valeur universelle exceptionnelle : le critère (iii) parle de la valeur de témoignage d'une culture, le critère (v) concerne le rapport de l'homme à son environnement et surtout le critère (vi) se rapporte aux traditions vivantes, aux idées, aux croyances et aux œuvres artistiques et littéraires. Au-delà de ces critères, le patrimoine immatériel repose sur une toute autre logique que celle de l'exceptionnalité du patrimoine mondial, en cherchant à représenter les différentes pratiques culturelles sans hiérarchie de valeur;

12 Voir Rapport final de l'Audit de la Stratégie globale et de l'initiative PACTe p. 25-29 (WHC-11/ 35.COM/INF.9A) et l'Annexe I (p. 68).

13 Ch. HotTin, Une nouvelle perception du patrimoine, op. cit., p. 15.

14 Suite à l'affaire El condor pasa en 1970 où la célèbre chanson de Paul Simon et Art Garfunkel fut perçue comme une exploitation illégitime de la musique indigène des Andes par les pays des Andes, le ministre des Affaires étrangères et de la Religion de la République de Bolivie adressa une lettre au directeur général de l'UNESCO constatant « l'exportation clandestine des plus intensives » des formes d'expression telles que la musique ou la danse et demandant trois nouvelles mesures dont l'une d'entre elles visait à la signature d'une nouvelle convention « pour réguler dans tous ses aspects la préservation, la promotion et la diffusion du folklore » assortie d'un « Registre international de la propriété culturelle du folklore», voir V. Tr. HALFSTEIN, Célébrer les différences, renforcer la conformité, dans Ch. BоRTOLOTTO (sous la dir.), Le patrimoine immatériel, op.cit. p. 75-77.

15 J. BLAKE, Élaboration d'un nouvel instrument normatif, op. cit., p. 78-79.

16 Paragraphe 12 des Principes et directives pour la protection du patrimoine des peuples autochtones (1995), établis par Mme Erica Irene DAES, rapporteur spécial de la Sous-Commission de la lutte contre les mesures discriminatoires et de la protection des minorités.

17 Outre de nombreuses déclarations depuis les années soixante-dix, la Décennie internationale des populations autochtones de 1993 à 2004 déboucha sur l'adoption de la Déclaration des Peuples autochtones et la protection internationale et nationale des connaissances traditionnelles du 19 juillet 2005, 23 ème session du Groupe de Travail sur le Droits des Peuples Autochtones, suivie de la Déclaration des Nations Unies sur les droits des peuples autochtones du 2 octobre 2007, A/RES/61/295.

18 C. MARTINEZ, La question des connaissances autochtones en droit international. Contenu ou fiction? Une problématique juridique de qualification et de protection, dans N. MEZGHANi et M. CORNU (sous la dir.), Intérêt culturel et mondialisation, les aspects internationaux, t. II, Paris, 
La norme protectrice du divertissement : le droit du patrimoine culturel imma...

L’Harmattan, 2004, p. 19-38 (Droit du patrimoine culturel et naturel).

19 Glossaire des principaux termes relatifs à la propriété intellectuelle et aux ressources génétiques, aux savoirs traditionnels et aux expressions culturelles traditionnelles, Comité intergouvernemental de la propriété intellectuelle relative aux ressources génétiques, aux savoirs traditionnels et au folklore, $21^{\text {ème }}$ session, Genève, 16-20 avril 2012, WIPO/GRTKF/IC/21/INF/8, Annexe, p. 39-40.

$\underline{20}$ Il s'agit du mode de vie, pris dans son ensemble, d’une société donnée, intégrant des aspects tels que les techniques et le savoir-faire, la langue, les valeurs, les cérémonies et les rites, les croyances religieuses et spirituelles, les symboles et les relations entre les sexes.

21 J. BLAKE, Élaboration d'un nouvel instrument normatif, op. cit., p. 54.

$\underline{22}$ Idem, p. 53.

23 Convention des Nations Unies sur la biodiversité de 1992, qui est - mis à part la Convention de 2003 - le seul instrument international juridiquement contraignant qui fait explicitement mention de la protection des connaissances traditionnelles.

$\underline{24}$ «Sous réserve des dispositions de sa législation nationale, respecte, préserve et maintient les connaissances, innovations et pratiques des communautés autochtones et locales qui incarnent des modes de vie traditionnels présentant un intérêt pour la conservation et l'utilisation durable de la diversité biologique et en favorise l'application sur une plus grande échelle, avec l'accord et la participation des dépositaires de ces connaissances, innovations et pratiques et encourage le partage équitable des avantages découlant de l'utilisation de ces connaissances, innovations et pratiques », article 8, j) de la Convention sur la biodiversité de 1992.

$\underline{25}$ J. BLAKE, Élaboration d'un nouvel instrument normatif, op. cit., p. 66, n. 325.

26 Premier considérant du Préambule de la Convention de 2003.

27 Préambule Convention, alinéa 3 et 2 .

$\underline{28}$ J. BLAKE, Élaboration d'un nouvel instrument normatif, op. cit., p. 3.

29 P. SAUVÉ, Document de réflexion sur : Le traitement des produits et services culturels dans les accords commerciaux. Deuxième Concertation intergouvernementale, Paris le 12 décembre 2000. Thème : La promotion de la diversité culturelle, Cambridge, Center for Business and Government, Université Harvard, octobre 2000, p. 3.

30 J. BLAKE, Élaboration d'un nouvel instrument normatif, op. cit., résumé analytique.

$\underline{31}$ Recommandation sur la sauvegarde de la culture traditionnelle et populaire, section A.

32 J. BLAKE, Élaboration d'un nouvel instrument normatif, op. cit., p. 36-37.

$\underline{33}$ Recommandation sur la sauvegarde de la culture traditionnelle et populaire, section C.

34 J. BLAKE, Élaboration d'un nouvel instrument normatif, op. cit., p. 42.

$\underline{35}$ Idem, p. 47. 
36 R. SMEETS, Une convention internationale. Une convention passe aux actes : Organisation, directives, calendrier, dans Ch. HotTIN (coordonnateur), Le patrimoine culturel immatériel, op.cit. p. 12. Il y a toutefois lieu de relever l'absence de ratification des pays anglo-saxons.

37 Directives opérationnelles suite aux révisions de la $4{ }^{\text {ème }}$ AG (4-8 juin 2012) :

38 T. ScovazzI, Le patrimoine culturel de l'humanité - Bilan de recherches de la section de langue française, dans Le patrimoine culturel de l'humanité, La Haye, Académie internationale de La Haye, 2005, p. 124.

39 Ch. HotTin, Une nouvelle perception du patrimoine, op. cit., p. 17.

40 T. ScovazZI, Le patrimoine culturel, op. cit., p. 127.

41 Article 1 (a) et 19, § 2 de la Convention de 2003.

$\underline{42}$ La tenue et mise à jour d'inventaires (art. 12), l'adoption d'autres mesures de sauvegarde, de développement et de mise en valeur de ce patrimoine (art. 13), la promotion de programmes d'éducation, ainsi que la sensibilisation et le renforcement des capacités (art. 14).

43 L'échange d'informations et d'expériences, les initiatives communes ainsi que la mise en place d'un mécanisme d'assistance aux États parties dans leurs efforts pour sauvegarder le patrimoine culturel immatériel (art. 19).

44 T. ScovazzI, Le patrimoine culturel de l'humanité, op. cit., p. 129.

$4 \underline{5}$ Protection des savoirs traditionnels et propriété intellectuelle dans les pays en développement : rapport de situation, Comité permanent de la coopération pour le développement en rapport avec la propriété intellectuelle, $2^{\text {ème }}$ session, 5-8 février 2001, PCIPD/2/7, p. 2.

46 Bien que le thème des ressources génétiques est lié aux deux autres, il ne fait néanmoins pas partie du patrimoine culturel immatériel, mais est analysé conjointement avec les deux autres thèmes dans l'OMPI.

47 L. WANG, La convention, op. cit., p. 175-177.

48 Création de l'IGC lors de la $26^{\text {ème }}$ session de l'Assemblée générale des États membres de l’OMPI, tenue à Genève du 25 septembre au 3 octobre 2000 (WO/GA/26/6).

49 A savoir les questions de la propriété intellectuelle que soulèvent a) l'accès aux ressources génétiques et le partage des avantages qui en découlent; b) la protection des savoirs traditionnels, associés ou non à ces ressources ; et c) la protection des expressions du folklore.

$\underline{50}$ http://www.wipo.int/tk/fr/igc/, consulté le 31 mars 2015.

51 B. FAVEL, Les problématiques européennes, dansCh. HotTIN (coordonnateur), « Le patrimoine culturel immatériel », op.cit., p. 22.

52 Article 167 du Traité sur la Fonctionnement de l’Union Européenne (TFUE) : «1. L’Union contribue à l'épanouissement des cultures des États membres dans le respect de leur diversité nationale et régionale, tout en mettant en évidence l’héritage culturel commun. 
La norme protectrice du divertissement : le droit du patrimoine culturel imma...

53 Décision n 1194/2011/UE du Parlement européen et du Conseil du 16 novembre 2011 établissant une action de l'Union européenne pour le label du patrimoine européen (http://eurlex.europa.eu/LexUriServ/LexUriServ.do?uri=OJ:L:2011:303:0001:0009:EN:PDF, consulté le 2 avril 2015).

$\underline{54}$ Article 2 de la décision n 1194/2011/UE : «Aux fins de la présente décision, on entend par :

55 Avis du Comité des régions sur le «Label du patrimoine européen », 85e session plénière des 9 et 10 juin 2010 (2010/C 267/11).

56 Il s'agit de : Carnuntum Archaeological Park, un quartier reconstruit d'une cité romaine dans le Bad Deutsch-Altenburg, en Autriche ; la maison médiévale des guildes de Tallinn, en Estonie (Great Guild Hall) ; le palais centenaire de la paix à la Haye (Peace Palace) et le Camp Westerbork, un camp de transit nazi pendant la Deuxième Guerre mondiale, situé à Hooghalen, également au Pays-Bas ; voir le communiqué de presse à ce sujet, http://europa.eu/rapid/pressrelease_IP-13-1177_fr.htm, consulté le 2 avril 2015.

57 La Convention de culture européenne (1954), la Convention pour la sauvegarde du patrimoine architectural de l'Europe (1985), la Convention européenne pour la protection du patrimoine archéologique (1992, révisée) et la Convention européenne du paysage (2000).

58 La Convention tire son origine des travaux lancés par le Conseil de l'Europe sur le thème « Patrimoine, identité et diversité » après la $4^{\mathrm{e}}$ Conférence des Ministres responsables du patrimoine culturel (Helsinki, mai 1996) venus répondre aux destructions constatées d'éléments du patrimoine culturel lors de conflits récents survenus en Europe, Rapport explicatif de la Convention-cadre du Conseil de l'Europe sur la valeur du patrimoine culturel pour la société (http://conventions.coe.int/Treaty/FR/Reports/Html/199.htm), consulté le 3 avril 2015.

59 J. Fromageau, Droit et patrimoine (évolution de la notion), dans Actes du colloque «Le patrimoine oui, mais quel patrimoine » organisé par la Commission Nationale Française pour l'UNESCO avec la collaboration de la MCM/Centre français du patrimoine culturel immatériel, 3 et 4 avril 2012, UNESCO, Paris.

60 Préambule de la Convention-cadre sur la valeur du patrimoine culturel pour la société.

61 M. JACOBS, Fracture culturelle, héritage des communautés et programmes de sauvegarde, dans Actes de la $9^{\text {ème }}$ Journée du patrimoine culturel immatériel, Premier Forum de chercheurs du $P C I$, organisée par la Maison des Cultures du Monde, le Centre français du patrimoine culturel immatériel et L'international Research Centre for Intangible Cultural Heritage in Asia and the Pacific Region (IRCI), le 3 juin 2012, Maison des Cultures du Monde, Paris.

62 La communauté du patrimoine est une communauté qui «se compose de personnes qui attachent de la valeur à des aspects spécifiques du patrimoine culturel qu'elles souhaitent, dans le cadre de l'action publique, maintenir et transmettre aux générations futures », article 2 (b) de la Convention-cadre.

63 Article 4 (a) de la Convention-cadre : " toute personne, seule ou en commun, a le droit de bénéficier du patrimoine culturel et de contribuer à son enrichissement ; ».

$\underline{64}$ http://www.federation-wallonie-bruxelles.be/index.php?id=2394, consulté le 3 avril 2015. 
65 Articles 1 à 3 de la Constitution belge. La répartition s'opérant sur des bases territoriales et non personnelles comme pour les communautés, divisant ainsi le territoire belge en la Région wallonne, la Région de Bruxelles-Capitale et la Région flamande.

66 F. MAguet, L'image des communautés dans l'espace public, dans Ch. BortolotTo (sous la dir.), Le patrimoine immatériel, op.cit., p. 55-56.

67 C.A., arrêt n 24/94 du 10 mars 1994, B.4.

68 C.C., arrêt n² 25/2010 du 17 mars 2010, B.4.1. se référant aux Doc. Parl., Sén., sess. ord. 1970-1971, n 400, p. 4-5.

69 Exposé des motifs de la loi du 21 juillet 1971, Pasin., 1971, p. 1461 cité par M. QuINTIN, La protection du patrimoine culturel, Brugge, Vanden Broele, 2009, p. 32.

70 Article $6, \S 1^{\mathrm{er}}$, I, $7^{\circ}$ de la Loi spéciale du 8 août 1980 tel qu'inséré par l'article 4 , § $1^{\mathrm{er}}$ de la Loi spéciale du 8 août 1988, M.B., 13 août 1988, en vigueur le $1^{\text {er }}$ janvier 1989.

71 A.-M. DRAYE, De zorg voor monumenten en landschappen in de notariële praktijk, juridische aspecten, dans Het milieu - L'enironnement, p. 72 cité par M. QUINTIN, op. cit., p. 34.

72 - Article 6bis, § 2, $4^{\circ}$ de la Loi spéciale du 8 août 1980, remplacé par l'article 3, § 2, de la Loi spéciale du 16 juillet 1993 visant à achever la structure fédérale de l’État, M.B., 20 juillet 1993 ;

73 A.R. du 30 octobre 1996 désignant les établissements scientifiques et culturels fédéraux, M.B., 7 décembre 1996, modifié par l’A.R. du 9 avril 2007, M.B., 20 avril 2007 reprenant les établissements suivants : les archives générales du Royaume et les archives de l'État dans les provinces, la Bibliothèque royale de Belgique, l’Institut d'Aéronomie spatiale de Belgique, l'Institut royal des Sciences naturelles de Belgique, l'Institut royal du Patrimoine artistique, l’Institut royal de météorologie de Belgique, le Musée royal de l’Afrique centrale, les Musées royaux d'Art et d'Histoire, les musées royaux des Beaux-Arts, l'Observatoire royal de Belgique, le centre d'études et de documentation Guerre et sociétés contemporaines, centre autonome rattaché aux Archives générales du Royaume et à la Bibliothèque royale de Belgique.

74 B. Genest, Le Patrimoine immatériel en Belgique francophone : un modèle pour le Québec, dans Rabaska : revue d'ethnologie de l'Amérique française, vol. 5, 2007, p. 48, http://id.erudit.org/ iderudit/019025ar, consulté le 3 avril 2015.

75 Décret relatif aux biens culturels mobiliers et au patrimoine immatériel de la Communauté française du 11 juillet 2002, M.B., 24 septembre 2002.

76 Le Japon s'est doté d'un instrument législatif dès les années cinquante par sa loi sur la protection des biens culturels (1950) et constitue une des premières sources nationales dans le domaine, servant de modèle à de nombreux pays, dont l'entité fédérée belge,

http://www.unesco.org/culture/ich/index.php?cp=JP\&lg=fr\&pg=00311\&topic=lht, consulté le 3 avril 2015.

77 Voy. les propos tenus par le Ministre de la Culture de la Communauté française à l'époque, Mr Rudy Demotte, dans la revue Museum international : " J'ai voulu, en tant que ministre de la culture responsable de la protection du patrimoine culturel, étendre au patrimoine immatériel la 
La norme protectrice du divertissement : le droit du patrimoine culturel imma...

reconnaissance accordée au patrimoine culturel mobilier. Le patrimoine immatériel, c'est précisément l'ensemble des valeurs, des savoir-faire, mais aussi des émotions qui donnent un sentiment d'appartenance, d'où que l'on soit. (...) Convaincu par la cause défendue par l'UNESCO, j'ai décidé de transcrire en communauté française les mesures proposées par l'organisation internationale pour la défense de ce patrimoine et le décret de la communauté française relatif au patrimoine culturel dans son ensemble a été voté le 3 juillet 2002 », R. DEMOTTE, « Les politiques nationales en matière de patrimoine culturel immatériel : le cas de la communauté française de Belgique », Museum international, vol. 56, n 1-2, 2004, p. 175, http://unesdoc.unesco.org/images/ 0013/001358/135852fo.pdf, consulté le 29 septembre 2015.

78 B. GENEST, Le patrimoine, op. cit., p. 55.

79 L'expert de la Communauté française, Mr. Jean-Pierre DuCASTELLE, a étroitement participé à la rédaction et à l'adoption de la Convention de 2003, étant encore présent à la $4{ }^{\text {ème }}$ Assemblée Générale des États Parties à la Convention de 2003. L'expert flamand de l'époque, Marc JACOBS, fut mandaté par le directeur des bibliothèques publiques et l'éducation permanente des adultes pour participer à partir de 2001 aux réunions de l'UNESCO préparatoires à la Convention de 2003.

$\underline{80}$ Certains auteurs appellent au contraire à une modification du terme de " chef-d'œuvre », comme l'Unesco a elle-même procédé au changement, passant de chef-d'œuvre à « Liste représentative du patrimoine immatériel ». En effet, comme le note J.-P. DUCASTELLE, «L'appellation élitiste (de chef-d'œuvre) actuelle risque d'amener la commission à privilégier les grandes manifestations inscrites dans le calendrier touristique plutôt que des éléments authentiques discrets mais exprimant bien les attentes de leur communauté. Nous proposerons à la commission de reprendre la terminologie de l'Unesco qui correspond mieux à l'esprit de la convention. Le décret de 2002 devrait être modifié en ce sens». J.-P. DUCASTELLE, Le patrimoine culturel immatériel de la Fédération Wallonie-Bruxelles, dansS. САСНAT (dir.), L'administration du patrimoine culturel immatériel en Europe. Organisation, réglementation et procédures. Les Cahiers du CFPCI, n 1, 2013, p. 89, http://www.cfpci.fr/medias/site/Cahier_CFPCI_n1.pdf, consulté le 29 septembre 2015.

81 Article 26 du décret.

$\underline{82}$ Article 28 du décret.

83 Article 30 du décret.

$\underline{84}$ Article 15 de l'Arrêté du 4 septembre 2003 du Gouvernement de la Communauté française relatif aux titres de trésor culturel vivant et de chef- d'œuvre du patrimoine oral et immatériel et à l'octroi des subventions accordées aux personnes ayant reçu ce titre et aux opérateurs organisant les manifestations auxquelles ces titres ont été décernés, M.B., 11 mai 2004.

85 Article 2 de l'Arrêté du 4 septembre 2003 du Gouvernement de la Communauté française relatif aux titres de trésor culturel vivant et de chef- d'œuvre du patrimoine oral et immatériel et à l'octroi des subventions accordées aux personnes ayant reçu ce titre et aux opérateurs organisant les manifestations auxquelles ces titres ont été décernés, M.B., 11 mai 2004.

86 La composition de la Commission consultative du patrimoine oral et immatériel a été revue par 
l'Arrêté ministériel du 27 février 2013 nommant les membres de la Commission du patrimoine oral et immatériel ; voir le lien suivant : http://www.patrimoineculturel.cfwb.be/index.php?id=7298 , consulté le $1^{\mathrm{er}}$ avril 2015.

87 Arrêté du Gouvernement du 23 juin 2006 de la Communauté française instituant les missions, la composition et les aspects essentiels de fonctionnement d'instances d'avis tombant dans le champ d'application du décret du 10 avril 2003 relatif au fonctionnement des instances d'avis oeuvrant dans le secteur culturel, M.B., 27 septembre 2006 ; voir aussi l'Arrêté du Gouvernement de la Communauté française nommant les membres du Conseil d'ethnologie du 28 juin 2012, M.B., 5 octobre 2012.

88 Pour une liste complète, voir le lien suivant : http://www.patrimoineculturel.cfwb.be/ index.php?id=7301, consulté le $1^{\mathrm{er}}$ avril 2015.

89 J.-P. DuCASTELLE, Le Patrimoine Culturel Immatériel de la Fédération Wallonie-Bruxelles, dans Géants d'ici et d'ailleurs, janvier 2012, n 6-7, p. 13.

90 En 2013, $4.020 €$ de subsides ont été attribués, http://www.patrimoineculturel.cfwb.be/ index.php?id=9315, consulté le 3 avril 2015.

91 Décret du 23 mai 2008 portant développement, organisation et subventionnement de la politique flamande du patrimoine culturel, M.B., 8 avril 2008, révisé par le Décret du 6 juillet 2012 relatif à la politique flamande en matière de patrimoine culturel, M.B., 3 septembre 2012.

92 Article $2,1^{\circ}$ du décret du 23 mai 2008 portant développement, organisation et subventionnement de la politique flamande du patrimoine culturel précise que le patrimoine culturel se réfère aux « supports de signification matériels et immatériels du passé, qui acquièrent des références communes dans un cadre de référence culturel ».

$\underline{93}$ http://www.kunstenenerfgoed.be/sites/default/files/uploads/

140422_Reglement\%20Inventaris\%20Vlaanderen\%20voor\%20Immaterieel\%20Cultureel\%20Erfgoed\%20\%28PDF, consulté le 3 avril 2015.

94 La Communauté flamande a veillé à nommer cet inventaire, «Inventaire en Flandre » et non « Inventaire flamand » afin d'indiquer qu'il vise à représenter l'ensemble des pratiques, usages, traditions et savoir-faire présents sur le territoire flamand, montrant une identité multiple.

95 Un aperçu de ces organisations peut être trouvé sur le site web www.kunstenenerfgoed.be.

96 Le décret de 2008 reprend le concept de « communauté du patrimoine » de la Conventioncadre du Conseil de l'Europe (2005).

97 http://www.faronet.be/fr/faro-interface-flamande-pour-le-patrimoine-culturel-asbl, consulté le 3 avril 2015 .

98 Selon ses propres termes, tels que remplis dans le « Rapport sur la mise en œuvre de la Convention et sur l'état des éléments qui ont été inscrits sur la Liste représentative du patrimoine culturel immatériel de l'humanité, Formulaire ICH-10 », remis le 9 août 2013 et qui peut être retrouvé via le lien suivant : http://www.kunstenenerfgoed.be/sites/default/files/uploads/ 140807 Belgisch\%20rapport\%20over\%20de\%20voortgang\%20mbt\%20de\%20implementatie\%20van\%20de\%20co 
La norme protectrice du divertissement : le droit du patrimoine culturel imma...

ou via le site de l'Unesco : http://www.unesco.org/culture/ich/index.php?lg=fr\&pg=00460 , consulté le $1^{\mathrm{er}}$ avril 2015.

$\underline{99}$ http://www.kunstenerfgoed.be/ake/view/nl/

4189951-Visienota_immaterieel_cultureel_erfgoed_dec10.html, consulté le 3 avril 2015.

100 Voir le lien suivant (seulement en allemand) : http://www.dgkulturerbe.be/

desktopdefault.aspx/tabid-3566/6369 read-37768/, consulté le $1^{\text {er }}$ avril 2015.

101 Voir le lien suivant : http://www.dgkulturerbe.be/desktopdefault.aspx/tabid-3466/

6370_read-42433/ (consulté le $1^{\mathrm{er}}$ avril 2015) et la création d'un atlas des dialectes régionaux.

102 Voir le lien suivant : http://www.dgkulturerbe.be/desktopdefault.aspx/tabid-3906/ (consulté le $1^{\mathrm{er}}$ avril 2015) et la reconnaissance de la culture de la bière.

103 M. CORNU, Safeguarding Heritage : From Legal Rights over Objects to Legal Rights for Individuals and Communities?, présenté lors du colloque The Future of the Past : Memory, History and Cultural Heritage in the 21st Century, organisé par le Ioannou Centre for Research in Classical and Byzantine Studies, University of Oxford, le vendredi 27 avril 2012.

104 Préambule de la Convention-cadre du Conseil de l’Europe sur la valeur du patrimoine culturel pour la société.

105 F. Ost et M. VAN DE KeRchove, De la pyramide au réseau ? Pour une théorie dialectique du droit, Bruxelles, Publications des Facultés universitaires Saint-Louis, 2002, p. 16.

106 Voir à ce sujet la thèse de C. ROMAINVILLE, Le droit à la culture, une réalité juridique, Bruxelles, Bruylant, 2014.

107 P. Halley et G. DE LASsus Saint-Genies, Mondialisation des concepts en droit de l'environnement : affaiblissement ou renforcement de la préservation du patrimoine culturel immatériel ? Les peuples inuits de l'arctique canadien et la protection juridique de l'environnement, dansJ. MorAnd DEVILLER et J.-C. BENICHOT, Mondialisation et globalisation des concepts juridiques. L'exemple du droit de l'environnement, IRJS Editions, 2010, p. 335 (Mission de Recherche « Droit et Justice », tome 22).

108 C. MARTINEZ, op. cit., p. 69.

109 Ibidem, p. 75.

110 Voy. à ce sujet notamment la question de la transmission du patrimoine immatériel, analysée par F. LEMPEREUR, La transmission du patrimoine culturel immatériel : réflexion sur l'importance d'une médiation culturelle, (thèse de doctorat de l'Université de Liège), Liège, 2008, 415 pages (non publiée).

111 Encore récemment, en 2014, la culture du carillon a été inscrite par l'Unesco sur la liste des Meilleures pratiques de sauvegarde (article 18 de la Convention de l'Unesco de 2003), suite au dossier international introduit par les communautés flamande et française, http://www.unesco.org/ culture/ich/index.php?lg=fr\&pg=00011\&Art18=01017, consulté le 29 septembre 2015. 
C@hiers du CRHIDI 2406-4157 Vol. 38 - 2016, 284

PDF généré automatiquement le 2020-07-07 08:37:17

Url de l'article : https://popups.uliege.be:443/1370-2262/index.php?id=284 